Conclusion Lipid profile changes in HGSC may have considerable prognostic value for the disease after treatment. The signatures defined by our work may provide a basis for the development of prognostic tools and may predict the clinical course of HGSC patients.

This work was supported by RSF grant $\square$ 20-65-46014. Disclosures Nothing to discloser.

\section{CAN D-DIMERS AND FIBRINOGEN AID IN THE DIAGNOSIS OF OVARIAN CANCER?}

${ }^{1}$ Kate Mckendry, ${ }^{2}$ Sharon O'toole. 'St James's Hospital; ${ }^{2}$ Department of Obstetrics and Gynaecology; Trinity College Dublin

\subsection{6/ijgc-2020-ESGO.103}

Introduction/Background Ovarian cancer (OC) mortality rates remain high due to a lack of early predictive biomarkers. Elevated levels of fibrinogen and its end product D-dimers are found in OC compared to benign controls and can predict poor prognosis independent of venous thromboembolism. Limited studies examining fibrinogen and D-dimers separately alongside CA125 suggest they may be of use in differentiating benign from malignant disease however no clear conclusions have been drawn due to a lack of prospective trials. The aim of this study was to evaluate the performance of D-dimers and fibrinogen alongside established predictors of OC, either alone or in combination, compared to CA125 alone.

Methodology Pre-operative serum samples were collected from 296 patients undergoing primary debulking surgery for pelvic masses. Levels of CA125, D-dimers, fibrinogen, Human Epididymis Protein 4 (HE4), the Risk of Ovarian Malignancy Algorithm, and the Risk of Malignancy Index I and II were assessed and sensitivity and specificity calculated. Logistic regression models were fitted for each individual biomarker and for various biomarker combinations and an ROC comparison was performed.

Results The study consisted of 96 pre- and 200 post-menopausal women with 154 benign, 43 borderline, and 99 malignant cases. CA125, D-dimers and fibrinogen had sensitivities of $73 \%, 75 \%$ and $65.2 \%$ and specificities of $69.5 \%, 55.9 \%$ and $57.6 \%$, respectively. The combination of HE4 and fibrinogen had the highest ROC-AUC in the premenopausal group at $75.8 \%$ but no biomarker(s) reached statistical significance compared to CA125. In the postmenopausal group, two panels $(\mathrm{CA} 125+\mathrm{HE} 4+\mathrm{D}$-dimer + fibrinogen, and HE4 $+\mathrm{d}$ dimer + fibrinogen) were significantly different to CA125 on ROC analysis (both $\mathrm{p}=0.029$ ).

Conclusion As individual biomarkers, D-dimers and fibrinogen are both limited by poor specificity using general population cut-offs. Where these biomarkers may play a role in OC is as part of biomarker panels. We developed a highly accurate multivariable model including HE4, d-dimers and fibrinogen which improved the diagnostic accuracy of CA125 alone in postmenopausal women. In pre-menopausal women, fibrinogen may play a role alongside HE4 as a second-step test, when imaging is inconclusive and CA125 is positive, to increase specificity.

Disclosures None.

\section{RISK FACTORS FOR PREOPERATIVE COMPLICATIONS AND MANAGEMENT WITH ENHANCED RECOVERY AFTER PRIMARY SURGERY FOR WOMEN WITH EPITHELIAL OVARIAN CANCER IN A SINGLE CENTER OF CHINA}

${ }^{1}$ Ying Zhou, ${ }^{2}$ Chenchen Zhu, ${ }^{3}$ Zhen Shen, ${ }^{3}$ Yanhu Xie, ${ }^{4}$ Wei Zhang, ${ }^{3}$ Tianjiao Zhang, ${ }^{3} \mathrm{LI}$ Min, ${ }^{3}$ Weidong Zhao, ${ }^{3}$ Dadao Wu, ${ }^{5}$ Björn Nashan. ${ }^{1}$ Department of Obstetrics and Gynecology, The First Affiliated Hospital of Ustc, Division of Life Sciences and Medicine, University of Science and Technology of China; ${ }^{2}$ Department of Obstetrics and Gynecology, Anhui Provincial Hospital, Anhui Medical University; ${ }^{3}$ The First Affiliated Hospital of Ustc, Division of Life Sciences and Medicine, University of Science and Technology of China; Department of Obstetrics and Gynaecology; ${ }^{4}$ The First Affiliated Hospital of Ustc, Division of Life Sciences and Medicine, University of Science and Technology of China; Department of Anaesthesiology; ${ }^{5}$ The First Affiliated Hospital of Ustc, Division of Life Sciences and Medicine, University of Science and Technology of China; Organ Transplantation Center

10.1136/ijgc-2020-ESG0.104

Introduction/Background This study aimed to evaluate the postoperative complications and their impacts on patients who have undergone primary surgery (including extensive upper abdominal surgery) of ovarian epithelial cancer with the enhanced recovery programme.

Methodology We identified all patients with stage I $\square$ IV ovarian carcinoma who underwent primary surgery in our centre. Postoperative complications were evaluated and graded according to the Clavien-Dindo Classification.

Results Among 161 patients, 46 cases (28.57\%) were performed with surgical staging, $27(16.77 \%)$ with standard cytoreduction, $12(7.45 \%)$ with en-bloc debulking, and 76(47.20\%) with extraradical debulking; 157 patients (97.52\%) achieved optimal tumour reduction $(<1 \mathrm{~cm})$. The mean postoperative hospitalisation time was $17.33 \pm 11.29$ days after completion of initial postoperative chemotherapy (IPC), and the interval of IPC was $16.22 \pm 10.09$ days. Thirteen patients $(8.07 \%)$ had grade 3 complications (9 wound dehiscence, 3 digestive tract leakage, and 1 bladder fistula). Two patients (1.24\%) had grade 4-5 complications (1 severe pneumonia infection and back to intensive care unit [ICU] for tracheotomy and respiration rehabilitation; 1 died of septicaemia on day 19). As for preoperative factors analysis, multivariate analysis revealed that HE4 $\geq 717 \mathrm{pM}(\mathrm{P}=0.015)$ and Federation International of Gynecology and Obstetrics (FIGO) IV stage ( $\mathrm{P}=0.004$, compared with IIIC stage) were associated with grade $3 \square 5$ complications. Bootstrap analysis found CA125 $\geq 1012 \mathrm{U} / \mathrm{mL}(\mathrm{P}=$ $0.034)$, HE4 $\geq 717 \mathrm{pM}(\mathrm{P}=0.007)$, and FIGO IV stage ( $\mathrm{P}$ $=0.002$, compared with IIIC stage) had statistical significance. As for postoperative factors analysis, multivariate analysis did not reveal the risk factors associated with grade $3 \square 5$ complications; bootstrap analysis only found that transfer to ICU after surgery $(\mathrm{P}=0.026)$ had statistical significance.

Conclusion Application of enhanced recovery after surgery protocols in epithelial ovarian carcinoma are useful and support the early initiation of chemotherapy and a short hospitalisation time, and it is safe for primary extensive radical cytoreduction with low mortality $1 / 76$ (1.31\%).

Disclosures This study was funded by the National Natural Science Foundation of China (81872110, 81902632), National Key R\&D Program of China (2018YFC1003900), the Anhui Provincial Innovative Program for Organ Transplantation (S20183400001), and Anhui Provincial Key Research and Development Program (1704a0802151). The funders had no 